\title{
New constraints on the multiplicity of massive young stars in Upper Scorpius ${ }^{\star}, \star \star$
}

\author{
R. Grellmann ${ }^{1,4}$, Th. Ratzka ${ }^{1,5}$, R. Köhler ${ }^{2,3}$, Th. Preibisch $^{1}$, and P. Mucciarelli ${ }^{1}$ \\ ${ }^{1}$ Universitäts-Sternwarte München, Ludwig-Maximilians-Universität, Scheinerstr. 1, 81679 München, Germany \\ e-mail: grellman@usm.uni-muenchen.de \\ 2 Landessternwarte Heidelberg, Königstuhl 12, 69117 Heidelberg, Germany \\ 3 Max-Planck-Institut für Astronomie, Königstuhl 17, 69117 Heidelberg, Germany \\ ${ }^{4}$ European Southern Observatory, Alonso de Cordova 3107 Vitacura, Casilla 19001, Santiago de Chile, Chile \\ 5 Institute for Physics/IGAM, NAWI Graz, Karl-Franzens-Universität, Universitätsplatz 5/II, 8010 Graz, Austria
}

Received 11 May 2012 / Accepted 27 March 2015

\begin{abstract}
Context. Observations and simulations have clearly established that most stars form in multiple systems. Characterizing their properties is thus important for our understanding of the star formation process.

Aims. To provide statistics about the number of companions per star over the full range of angular distances, infrared long-baseline interferometric studies can be employed to fill the gap between spectroscopic and adaptive optics searches. The Upper Scorpius OB association is a good target for such observations, because its stellar content is very well known from both spectroscopic and adaptive optics searches.

Methods. We used the ESO Very Large Telescope Interferometer to perform long-baseline interferometric observations of a sample of seven B stars. Furthermore, we used ROSAT X-ray data to search for indications of low-mass companions.

Results. With the interferometric observations, we find previously known companions around $\sigma$ Sco and HR 6027. For the other targets we determine the parameter space in which the presence of companions can be excluded from our data. For two of the B stars in our sample, $\pi$ Sco and HR 6026, the detection of X-ray emission provides indirect evidence of previously unknown low-mass companions.

Conclusions. In total we find two previously unknown companions. We can exclude the presence of other unknown companions within the separation range of $\sim 2$ to $\sim 100$ mas and for a brightness ratio $\geq 0.1$.
\end{abstract}

Key words. techniques: interferometric - binaries: general - X-rays: stars - binaries : close - stars: formation - stars: massive

\section{Introduction}

An accurate characterization of multiple stellar systems and their properties is one of the most important steps in understanding the star formation process itself. Observations of star forming regions and young stellar clusters have clearly established that most, if not all, stars form in binaries or higher order multiple systems (e.g., Mathieu \& Zinnecker 2000). Stars with higher masses are found to be in multiple systems more often than low-mass stars (Delgado-Donate et al. 2004; Bate 2009; Preibisch et al. 2001). Furthermore, massive stars are often found in higher order multiple systems, i.e., triple or quadruple systems (Zinnecker \& Yorke 2007) and short-period binaries (Duchêne \& Kraus 2013). The observed properties of multiple systems, such as their separation, distribution, and mass ratios, provide important and strong constraints on star formation theories. During recent years, numerical simulations of star cluster formation have reached a level where they can make quite detailed predictions about the multiplicity of the forming stars (Goodwin et al. 2007; Bate 2009). To compare observational

* Based on observations collected at the European Organisation for Astronomical Research in the Southern Hemisphere, Chile, observing program 085.C-0260.

$\star \star$ Appendix $\mathrm{A}$ is available in electronic form at http://www. aanda.org surveys in a meaningful way, a complete knowledge of this primordial multiplicity in a well characterized sample of young stars is needed ${ }^{1}$

No single observational technique is able to reveal companions over the full range of possible separations. Spectroscopic studies can only find very close companions at separations of about one AU or less. For objects with distances of a few $100 \mathrm{pc}$, direct imaging (even with adaptive optics) can only reveal the wide companions at separations of more than tens or hundreds of AUs. Infrared long-baseline interferometry is very efficient in finding companions at angular separations between $\sim 2$ and $\sim 100$ mas. It is thus ideally suited to filling the observational gap between the very close spectroscopic companions and the wide visual companions.

Another (and completely independent) indication of a companion to a B-type star is the detection of X-ray emission. Stellar $\mathrm{X}$-ray emission (see Güdel \& Nazé 2009 for a recent review) is thought to be related either to coronal magnetic activity (e.g., Favata \& Micela 2003) for low-mass stars $\left(M<2 M_{\odot}\right)$ or to shocks in the wind of a very luminous O-type star (e.g., Kudritzki \& Puls 2000). For stars in the spectral range between

\footnotetext{
1 Complete means that all companion stars over the full range of possible orbital distances (extending from a few stellar radii to several 1000 AUs) are known or that companions in this range can be excluded with high confidence, respectively.
} 
Table 1. AMBER observations of targets in Upper Scorpius.

\begin{tabular}{|c|c|c|c|c|c|c|c|c|c|}
\hline \multirow{2}{*}{$\begin{array}{l}\text { Target } \\
\pi \mathrm{Sco}\end{array}$} & \multirow{2}{*}{$\begin{array}{c}\begin{array}{c}\text { Other } \\
\text { name }\end{array} \\
\text { HIP } 78265\end{array}$} & \multirow{2}{*}{$\begin{array}{c}\text { Date } \\
\text { May 06, } 2010\end{array}$} & \multicolumn{6}{|c|}{ Projected baselines } & \multirow{2}{*}{$\begin{array}{l}\text { Calibrator(s) } \\
\text { HD } 143900\end{array}$} \\
\hline & & & $87 \mathrm{~m}$ & $-88^{\circ}$ & $75 \mathrm{~m}$ & $151^{\circ}$ & $80 \mathrm{~m}$ & $-140^{\circ}$ & \\
\hline & & May 05,2010 & $90 \mathrm{~m}$ & $-149^{\circ}$ & $90 \mathrm{~m}$ & $-59^{\circ}$ & $126 \mathrm{~m}$ & $-104^{\circ}$ & HD 143404 \\
\hline$\tau$ Sco & HIP 81266 & May 10,2010 & $88 \mathrm{~m}$ & $-85^{\circ}$ & $76 \mathrm{~m}$ & $152^{\circ}$ & $79 \mathrm{~m}$ & $-139^{\circ}$ & HD 143404 \\
\hline$\rho \mathrm{Sco}^{*}$ & HIP 78104 & May 06, 2010 & $90 \mathrm{~m}$ & $-160^{\circ}$ & $88 \mathrm{~m}$ & $-70^{\circ}$ & $126 \mathrm{~m}$ & $-115^{\circ}$ & - \\
\hline$\sigma$ Sco & HIP 80112 & May 11,2010 & $124 \mathrm{~m}$ & $-117^{\circ}$ & $87 \mathrm{~m}$ & $109^{\circ}$ & $90 \mathrm{~m}$ & $-162^{\circ}$ & HD 135758, HD 143033 \\
\hline$\omega \mathrm{Sco}$ & HIP 78933 & May 11,2010 & $128 \mathrm{~m}$ & $-109^{\circ}$ & $91 \mathrm{~m}$ & $116^{\circ}$ & $90 \mathrm{~m}$ & $-154^{\circ}$ & HD 143033 \\
\hline \multirow[t]{2}{*}{$\chi \mathrm{Oph}$} & HIP 80569 & May 06, 2010 & $90 \mathrm{~m}$ & $-156^{\circ}$ & $90 \mathrm{~m}$ & $-66^{\circ}$ & $127 \mathrm{~m}$ & $-111^{\circ}$ & HD 135758 \\
\hline & & May 11, 2010 & $97 \mathrm{~m}$ & $-97^{\circ}$ & $72 \mathrm{~m}$ & $142^{\circ}$ & $86 \mathrm{~m}$ & $-142^{\circ}$ & HD 143033 \\
\hline HR $6027 \mathrm{~A} / \mathrm{C}$ & HIP 79374 & May 11,2010 & $128 \mathrm{~m}$ & $-106^{\circ}$ & $90 \mathrm{~m}$ & $119^{\circ}$ & $90 \mathrm{~m}$ & $-151^{\circ}$ & HD 143033 \\
\hline
\end{tabular}

Notes. ${ }^{(*)}$ Data of low quality and not further mentioned in discussion.

about B2 and A, neither of these two mechanisms can work (e.g., Schmitt et al. 1985; Preibisch et al. 2005). The detection of X-ray emission seeming to originate in a B-type star thus indicates the presence of a companion.

The goal of this paper is to present new constraints on the multiplicity of a brightness-selected sample of young stars in Upper Scorpius. The paper is organized as follows. In Sect. 2 we describe how and why the targets were selected. In Sect. 3 we briefly describe the interferometric data reduction and analysis strategy. Section 4 focuses on the data analysis and modeling. We finally discuss the analysis of archival X-ray data and their implications for the multiplicity in Upper Scorpius in Sect. 5.

\section{Target selection}

OB associations are well-suited to studies of stellar multiplicity, because their youth implies that the binary parameters of only a few of the most massive systems have changed and the low stellar densities guarantee that very little dynamical evolution has taken place. Moreover, in contrast to small clusters or T associations, like Taurus-Auriga, OB associations contain a much wider range of stellar masses, including high- and intermediate-mass stars.

With a distance of $145 \mathrm{pc}$ (de Zeeuw et al. 1999), the OB association nearest to the Sun is the Sco OB2 or ScorpiusCentaurus association. It consists of three subgroups, the youngest of which is Upper Scorpius (see e.g., Preibisch \& Mamajek 2008; Blaauw 1991). The star formation process in Upper Sco is finished and there are neither dense molecular clouds nor deeply embedded young stellar objects. An extensive (and definitive) investigation of the high- and intermediatemass stellar populations in Scorpius-Centaurus was carried out by de Zeeuw et al. (1999). They used HIPPARCos proper motions and parallaxes in conjunction with two moving group methods to accurately establish the stellar content down to mid-F spectral type $\left(\sim 2 M_{\odot}\right)$ and identified 120 stars as genuine members. In a series of spectroscopic studies, Preibisch \& Zinnecker (1999) and Preibisch et al. (2002) have identified a large and statistically complete sample of low-mass members and then investigated the stellar population and star formation history of Upper Scorpius over the full stellar mass range from $0.1 M_{\odot}$ to $20 M_{\odot}$. They found that the whole stellar population in Upper Sco is very well characterized by a narrow age distribution around $5 \mathrm{Myr}$ and that the initial mass function of Upper Sco is, within the uncertainties, consistent with recent determinations of the field initial mass function. These findings have been confirmed by an independent study by Slesnick et al. (2008).
Kouwenhoven et al. $(2005,2007)$ have published an extended study of the primordial binary population in Scorpius Centaurus. Kouwenhoven et al. (2005) performed an adaptive optics search observing all HIPPARCOS members of spectral types A and late B using the ESO $3.6 \mathrm{~m}$ telescope at La Silla. By combining this data with those from the literature about multiplicity in Upper Scorpius, they found that more than $80 \%$ of the B0-B3 type have one or more companions. However, this number drops to $60 \%$ for B4-B9 type HIPPARCos members. The multiplicity for stars with spectral types A and F decreases even more. Very recently, Lafrenière et al. (2014) have performed a multiplicity survey of 91 stars in Upper Scorpius with the Gemini North telescope and conclude that inside their detection limits the companion star fraction is 0.30 . However, they point out that their results for the most massive (7.5-16 $\left.M_{\odot}\right)$ stars should be interpreted carefully, since close companions, which are expected with a high frequency for high-mass stars, could fall below the inner separation limit.

To perform a search for close visual companions and to close the gap between the already existing spectroscopic and adaptive optics surveys, we selected all members of the Upper Scorpius association, which are bright enough to be observed with AMBER and the Auxiliary Telescopes (thus $H$ - and $K$-band magnitudes $\leq 5.5 \mathrm{mag}$ ). AMBER is very well suited to finding these companions, because in the near-infrared the contrast between the bright B stars and secondary components is much less than in the optical. A similar approach has recently been followed by Rizzuto et al. (2013) who obtained interferometric observations between 550 and $800 \mathrm{~nm}$ with the Sydney University Stellar Interferometer (SUSI) of all stars in Sco-Cen that were brighter than 5 mag in the visual.

\section{Interferometric AMBER observations and data reduction}

The AMBER observations of B stars in Upper Scorpius at the VLTI were performed in May 2010 in the course of the ESO observing program 085.C-0260(A). AMBER (Petrov et al. 2007) is a multiaxial beam combiner combining light in the near infrared. Data were taken with three Auxiliary Telescopes (diameter of $1.8 \mathrm{~m}$ ) in the configuration $\mathrm{A} 0-\mathrm{G} 1-\mathrm{K} 0$ and in lowresolution mode (spectral resolution 35 ).

An overview of the performed AMBER observations, including projected baselines, position angles, and calibrators used, can be found in Table 1. In total, only seven B stars of the 14 sample stars were observed, five of them only once, and only the remaining two have been observed two times (as was 
Table 2. Companions of the USco targets.

\begin{tabular}{|c|c|c|c|c|c|c|}
\hline Star & Component & $\begin{array}{c}\text { Sep./period } \\
{\left[{ }^{\prime \prime}\right] / \text { days }}\end{array}$ & $\begin{array}{c}\text { Bright. }(V) \\
{[\mathrm{mag}]}\end{array}$ & $\mathrm{SpT}$ & $\begin{array}{l}\text { Mass } \\
{\left[M_{\odot}\right]}\end{array}$ & Reference/comment \\
\hline \multirow[t]{2}{*}{$\rho \mathrm{Sco}$} & $\mathrm{Aa}$ & & 3.81 & B2 & 10 & 2 \\
\hline & $\mathrm{Ab}$ & $4.003 \mathrm{~d}$ & & & & 2,5 \\
\hline \multirow[t]{3}{*}{$\pi \mathrm{Sco}$} & $\mathrm{Aa}$ & & 2.83 & B1 & 15 & 2 \\
\hline & $\mathrm{Ab}$ & $1.57 \mathrm{~d}$ & & B2 & 10 & 2,5 eclipsing binary \\
\hline & B & & & & 0.4 & see Sect. 5 \\
\hline \multirow[t]{2}{*}{$\tau \mathrm{Sco}$} & A & & 2.74 & B0 & 18 & 2 \\
\hline & B & 70.93 & 5.7 & & & 10 \\
\hline \multirow[t]{4}{*}{$\sigma \mathrm{Sco}$} & $\mathrm{Aa}$ & & 3.3 & B1 & 18.4 & 1,4 \\
\hline & $\mathrm{Ab}$ & $0.003^{\prime \prime}$ & 5.3 & B1 & 11.9 & 1,4 , see Sect. 4 \\
\hline & B & $0.43^{\prime \prime}$ & 5.2 & B7 & 3.5 & 1,4 \\
\hline & $\mathrm{C}$ & $20^{\prime \prime}$ & 8.6 & B9.5 & 2.7 & 4 \\
\hline$\omega$ Sco & A & & 3.93 & B1 & 15 & 2 \\
\hline \multirow[t]{3}{*}{$\chi \mathrm{Oph}$} & $\mathrm{Aa}$ & & 4.29 & B2 & 10 & 2 \\
\hline & $\mathrm{Ab}$ & $34.12 \mathrm{~d}$ & & & & 2,3 \\
\hline & Ac & $138.8 \mathrm{~d}$ & & & & 5 \\
\hline \multirow[t]{8}{*}{$v \mathrm{Sco}$} & HR $6027 \mathrm{Aa}$ & & 4.37 & B2IV & 10 & 1 \\
\hline & HR $6027 \mathrm{Ab}$ & $5.55 \mathrm{~d}$ & 6.9 & & 1 & $1,5,6,7$ \\
\hline & HR 6027 B & $1.305^{\prime \prime}$ & 5.4 & & 6 & $1,5,6,10$ \\
\hline & HR 6027 C & $0.063^{\prime \prime}$ & 6.6 & & 6 & 1,8, Sect. 4 \\
\hline & HR 6026 A & $41.1^{\prime \prime}$ & 6.9 & B8V & 3 & 1 \\
\hline & HR $6026 \mathrm{Ba}$ & $2.0^{\prime \prime}$ & 7.4 & B9V & 2.7 & 1,6 \\
\hline & HR $6026 \mathrm{Bb}$ & & 7.9 & B9V & 2.7 & 1,9 \\
\hline & HR 6026 C & & & & 1.5 & see Sect. 5 \\
\hline
\end{tabular}

References. (1) MSC, Tokovinin (1997); (2) BSC, Eggleton \& Tokovinin (2008); (3) Harmanec (1987); (4) North et al. (2007); (5) Kouwenhoven et al. (2007); (6) Levato et al. (1987); (7) Batten et al. (1989); (8) McAlister et al. (1990); (9) Andersen \& Nordstrom (1983); (10) Lafrenière et al. (2014).

planned for the other targets as well). However, the data taken of $\rho$ Sco are very noisy and thus not taken into account for the further analysis and discussion. More information on the stellar properties and multiplicity of all targets can be found in Table 2 .

For the data reduction and calibration amdlib Vers. 3.0.4 (Tatulli et al. 2007; Chelli et al. 2009) provided by the JeanMarie-Mariotti-Center ${ }^{2}$ was used. The data was calibrated by using only the associated calibrator. This is a simplified method, assuming that the (instrumental and atmospheric) transfer function has been stable between the science target and the calibrator.

In a first approximation, a binary can be described as composition of two sources separated by the distance $a$. Because the radius for a star of spectral type $\mathrm{B} 1$ is $\sim 8 R_{\odot}$, corresponding to a size $\sim 0.25$ mas, the stars themselves are practically unresolved in the AMBER observations. Thus, a model consisting of two point sources can be used. The squared visibility of a binary is given by

$V^{2}\left(B_{\lambda}\right)=\frac{1+f^{2}+2 f \cos 2 \pi \boldsymbol{a} \boldsymbol{B} / \lambda}{(1+f)^{2}}$,

where $\boldsymbol{a}$ is the separation vector in the plane of the sky (in radians), $\boldsymbol{B}$ the baseline vector joining the two telescopes (in meter), $\lambda$ the wavelength (in meters), and $f$ is the flux ratio of the two sources $\left(f=I_{1} / I_{2}\right)$. The frequency of this visibility oscillation depends on the separation of the components, whereas the amplitude depends on their flux ratio.

To define the area in the separation-position angle parameter space, where a binary can be excluded due to our AMBER data, we make the following assumption: we can identify a binary signal if we see at least half a period of the modulation in one band (i.e., $H$ - or $K$-band, sometimes we have only flux in

\footnotetext{
2 JMMC, http://www.jmmc.fr
}

the $K$-band data). The maximum separation is given by the interferometric field of view. Owing to the large errorbars of the visibility data, we would need a flux ratio $\gtrsim 0.1$ to detect a binary signal in the visibility curve. To check the sensitivity of the closure phase measurement we used ASPRO $^{3}$ to make models for different binary flux ratios. We find that for binaries with a flux ratio of $\sim 1: 15$ or less the closure phase is mostly $\leq 10^{\circ}$ (also depending on the separation of the components), and for a flux ratio of 1:10 the closure phase increases up to values between $10^{\circ}$ and $15^{\circ}$. Since our errors on the closure phase are usually between $5^{\circ}$ and $10^{\circ}$ we assume that this is the lowest flux ratio we would be able to detect from our closure phase measurement. The flux limits for a detection in the visibilities and a detection in the closure phase are thus similar. A global detection limit of 1:10 might appear coarse, but we checked for the sources without a companion above that limit that a single point source fits the data as well as a binary. Because a flux ratio of $1: 10$ corresponds to a magnitude difference of 2.5 we can, for instance, only detect companions with masses down to $\sim 1.7 M_{\odot}$ (Siess et al. 2000) to a primary of spectral type $\sim \mathrm{B} 3\left(M \sim 7 M_{\odot}\right)$.

\section{Interferometric results}

For $\sigma$ Sco we see a strong closure-phase signal and also a change in the visibility level (see Fig. 1). To model these signals we used the LitPRO tool developed at the Jean-Marie Mariotti Center (Tallon-Bosc et al. 2008). We find a companion located at -3.1 mas in right ascension and 0.08 mas in declination with respect to the primary (reduced $\chi^{2}=0.12$ ). The flux ratio of the two components is $0.5 \pm 0.1$. This companion is identical with an already known companion for which North et al. (2007) find a separation of 3.62 mas.

3 http://www.jmmc.fr/aspro_page.htm 

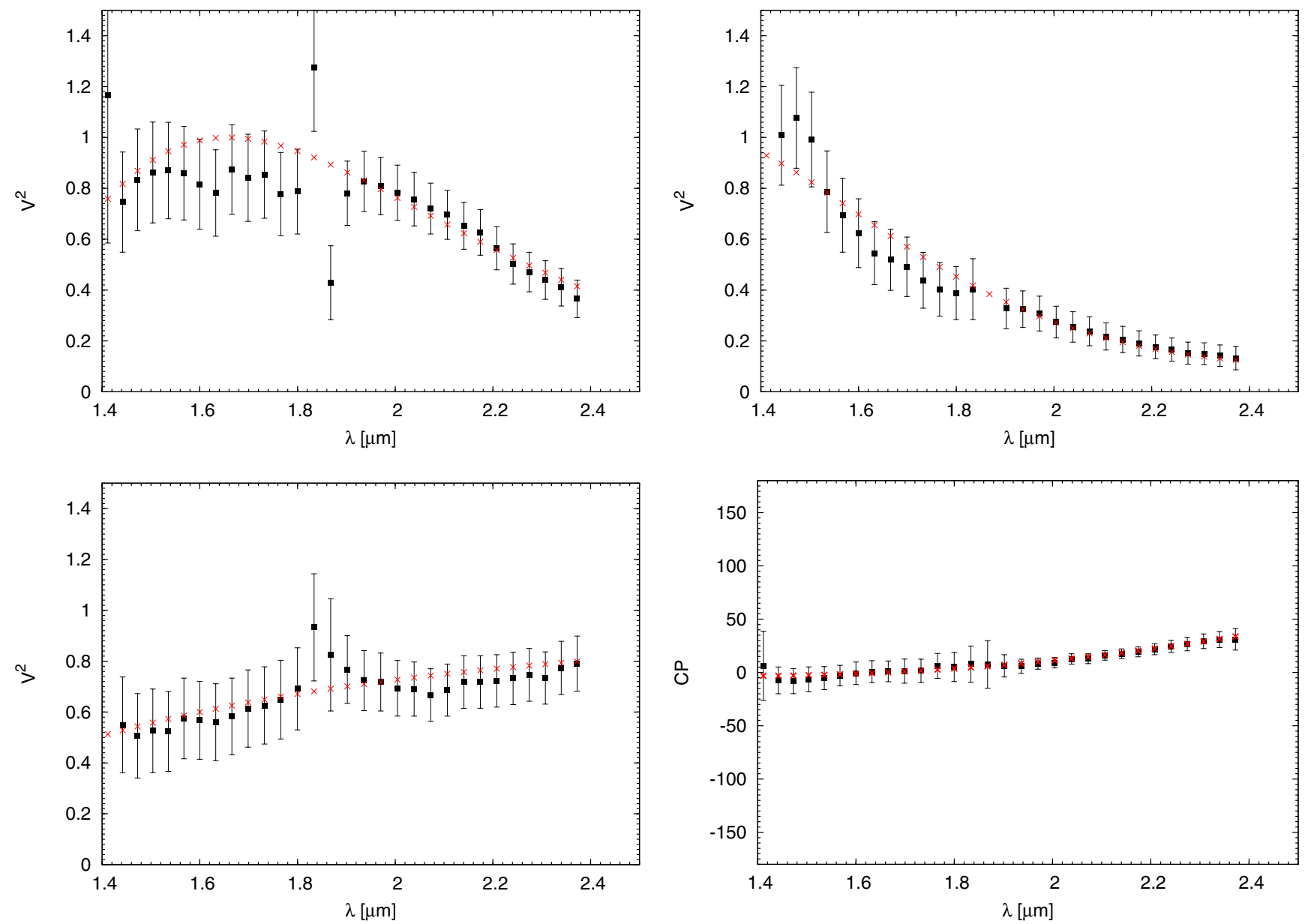

Fig. 1. Visibilities and closure phase of $\sigma$ Sco versus wavelength for the $H$ - and $K$-band measured with AMBER on 11/05/10 (black rectangulars). The LITpro binary model is shown with red crosses.

Also for HR 6027 we clearly detect a binary signal in the AMBER data, originating in the previously known companion HR 6027 C. In Fig. 2 the calibrated AMBER visibilities in the $H$ and $K$ bands are shown.

HR 6027 is a subsystem of the object $v$ Sco (HIP 79374, HR 6027/HR 6026), listed as a septuple system in the Multiple Star Catalog (MSC, Tokovinin 1997). The system can be divided into two subsystems, centered on the primary stars HR 6027 and HR 6026, which have a separation of 41.1". HR 6027 is a spectroscopic binary and has two visual companions at $\approx 1.3^{\prime \prime}$ and $\approx 0.060^{\prime \prime}$. HR 6026 has one visual companion at a distance of $\approx 2.0^{\prime \prime}$, which itself is a spectroscopic binary (see also Fig. 4). More details about this septuple of stars can be found in Table A.1. With AMBER we only observed the HR 6027 system.

To determine the position of HR $6027 \mathrm{C}$, we again used the LitPRO modeling tool. We find that the companion is located northwest of the primary with an offset in right ascension of -17.7 mas and in declination of 70.0 mas $\left(\chi^{2}=21.9\right)$. The uncertainty of the position as determined by the model fit is $\sim 1$ mas in RA and Dec. Furthermore, the uncertainty in the wavelength calibration is an additional error source and contributes an uncertainty of $\sim 0.5$ mas. The total error is thus considered to be 1.5 mas in each direction. In the $\chi^{2}$ map shown in Fig. 3 we can see several other local minima distributed around the minimum with the lowest $\chi^{2}$. The $\chi^{2}$ for these minima are, however, around two times higher than for the lowest minimum. For the flux ratio of HR $6027 \mathrm{~A}$ and HR $6027 \mathrm{C}$ the fit delivers $0.25 \pm 0.1$. The position obtained for $\mathrm{HR} 6027 \mathrm{C}$ is in very good agreement with the position obtained by Lafrenière et al. (2014) using the adaptive optics data of Gemini North.

Four objects $(\tau \mathrm{Sco}, \pi \mathrm{Sco}, \omega \mathrm{Sco}, \chi \mathrm{Oph})$ do not show any sign for a companion in their visibilities, although Rizzuto et al. (2013) detect a companion around $\tau$ Sco with a separation of $\sim 0.071^{\prime \prime}$. However, the flux difference between this companion and the primary is $\sim 3$ mag in the optical and might thus be out of our detection range in the near infrared. We used the LITpro tool to model the data first with a single point source and then with a binary consisting of two point sources. A binary model with a $\chi^{2}$ comparable to that of the point source model can only be found for binaries with very low flux ratios $(0.034$ for $\pi \mathrm{Sco}, 0.08$ for $\tau \mathrm{Sco}, \approx 0$ for $\omega \mathrm{Sco}$, and 0.08 for $\chi \mathrm{Oph}$ ). We thus can conclude that these sources do not have additional companions within the detection limits given in Sect. 3. Plots of the areas where a companion can be excluded from the AMBER data according to our definition can be found in Fig. A.1. We can estimate probabilities of missing a companion if it is by chance somewhere in the gaps of the covered area. The probability that we miss a component around one of the targets is $\sim 10-20 \%$ for a radius of $\sim 0.12^{\prime \prime}$ around the primary star.

\section{X-ray emission as a tracer of late type companions}

X-ray emission from stars is thought to be related either to coronal magnetic activity (e.g., Favata \& Micela 2003) for low-mass 
R. Grellmann et al.: NIR-interferometry in Upper Scorpius
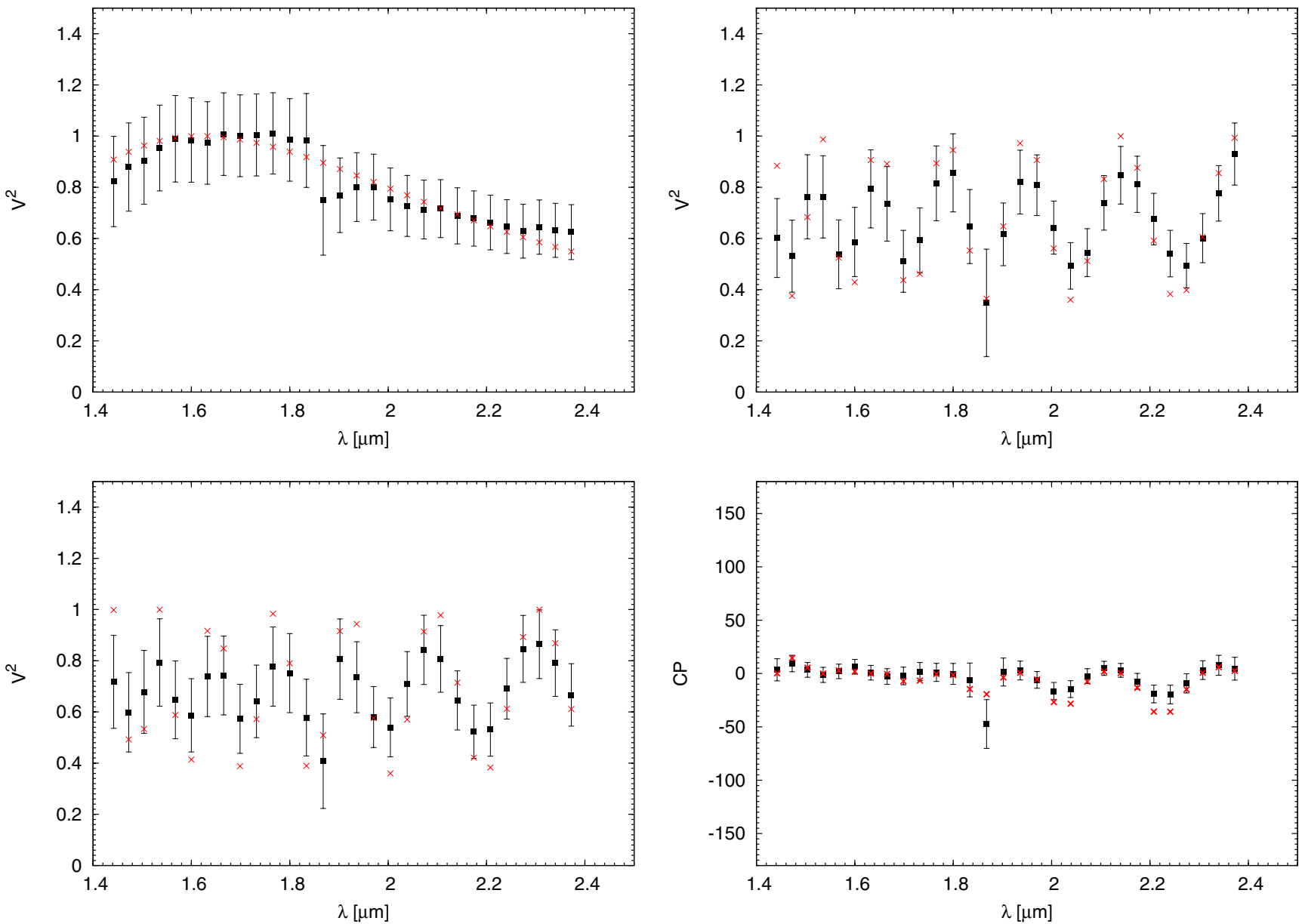

Fig. 2. Visibilities of HR 6027 versus wavelength for the $H$ and $K$ bands measured with AMBER (black rectangulars) together with the best-fit LitPRO model (red crosses). Upper left: visibility with a baseline of $128 \mathrm{~m}$ and a PA of $-106^{\circ}$. Upper right: visibility with a baseline of $90 \mathrm{~m}$ and a PA of $119^{\circ}$. Lower left: visibility with a baseline of $90 \mathrm{~m}$ and a PA of $-151^{\circ}$. Lower right: closure phase.

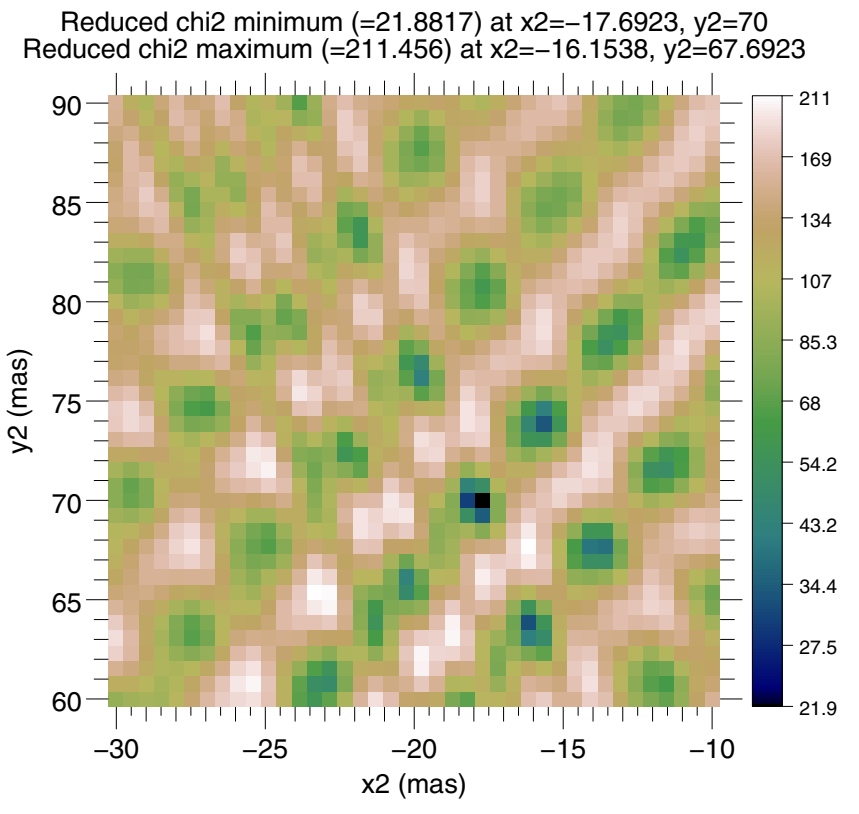

Fig. 3. Reduced $\chi^{2}$ map produced with LitPRO from the fit of HR 6027. Besides from the deepest minimum at -17.8 mas in RA and 70 mas in DEC we can see that there are other local minima.

stars $\left(M<2 M_{\odot}\right)$ or to shocks in the wind of the very luminous O-type star (e.g., Kudritzki \& Puls 2000). For stars with

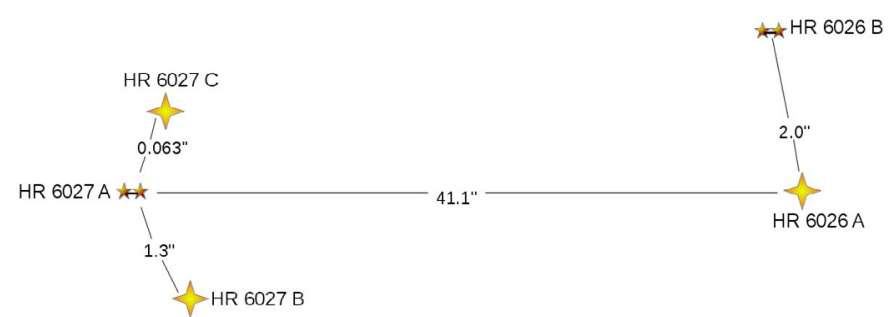

$\star \star$ Spectroscopic Binary

Fig. 4. Schematic view of the $v$ Sco system (not to scale).

spectral types between about B2 and A, neither of these two mechanisms can work: on the one hand, the winds of these stars are too weak to produce significant $\mathrm{X}$-ray emission in shocks; on the other hand, the lack of outer convection zones prevents the stellar dynamo generation of surface magnetic fields that would be required to produce significant X-ray emission from coronal plasma. These theoretical arguments are confirmed by the general lack of X-ray detections among late B- and A-type stars in a large number of X-ray observations (e.g., Preibisch et al. 2005; Stelzer et al. 2005).

Some late B (and A) stars have nevertheless been detected as rather strong X-ray sources (Stelzer et al. 2006). In several 


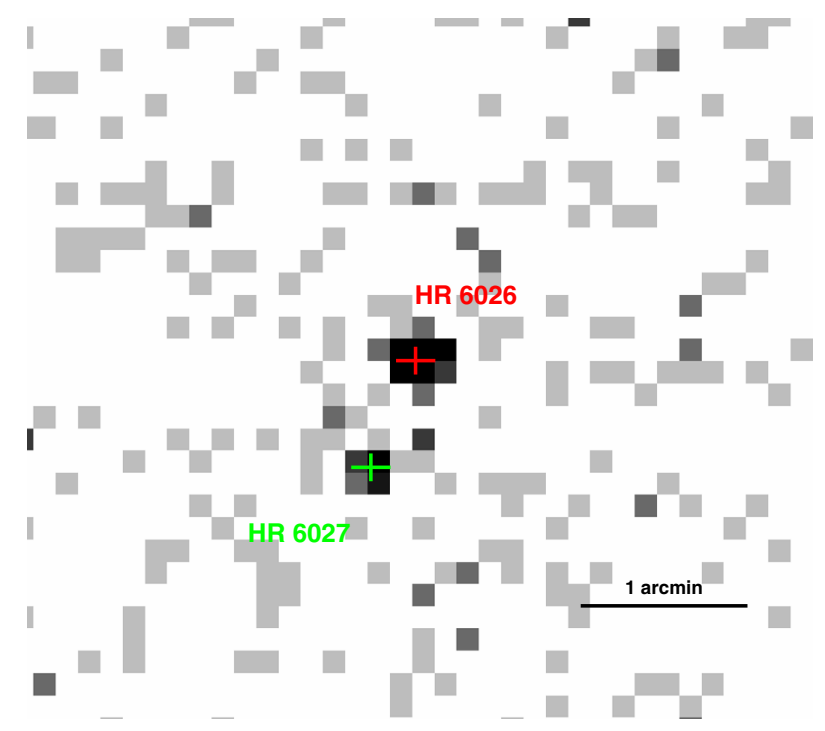

Fig. 5. ROSAT X-ray image of the $v$ Sco system.

of these cases, however, detailed observations (e.g., by the detection of X-ray eclipses) could show that the X-ray emission actually originates in a late-type (i.e., low-mass) companion and not from the B- or A-type primary star (e.g., Schmitt et al. 1993). In the context of the Chandra Carina Complex Project (Townsley et al. 2011), Evans et al. (2011) present evidence that the X-ray-detected late B stars are binaries with low-mass companions. Consequently, the detection of X-ray emission from a late B or an A-type star can be used as a good signature of an (unresolved) late-type companion.

We thus searched in the Heasarc Data Archive ${ }^{4}$ for X-ray observations of the selected stars. We found available ROSAT data for three sources: HR 6026/6027, $\pi$ Sco, and $\tau$ Sco. Count rates are extracted from circular regions for source and background using the FTOOLS XSELECT task. We then used WebPIMMS ${ }^{5}$ to convert the count rate into flux assuming a thermal APEC emission with $k T \sim 1 \mathrm{keV}$ and 0.4 solar abundances as typical of $\mathrm{T}$ Tauri stars. Galactic absorption is also taken into account using the $A_{V}$ values reported by de Bruijne (1999).

v Sco: (HR 6026/6027) these two close sources were observed by ROSAT with both HRI and PSPCB. We extracted the count rate from an on-axis HRI observation (Obs. Id. RH201631A01) that lasted about 2100 s. Extraction regions for HR 6026 and HR 6027 have radii of 20" to avoid contamination between the two sources. The background region has a $40^{\prime \prime}$ radius. The extracted net count rates are $(3.9 \pm 0.4) \times$ $10^{-2}$ counts s $^{-1}$ and $(1.2 \pm 0.3) \times 10^{-2}$ counts s $^{-1}$ for HR 6026 and HR 6027, respectively. The intrinsic luminosities for the two objects are $\log \left[L_{\mathrm{X}}\right] \sim 30.7$ and 30.2 , respectively (energy interval $[0.1-2.0] \mathrm{keV})$. According to the observationally established correlation between X-ray luminosity and stellar mass for coronal sources (see, e.g., Preibisch et al. 2005), these X-ray luminosities suggest companions with masses of $M \sim 1.5 M_{\odot}$ for the case of HR 6026 and $M \sim 1 M_{\odot}$ for HR 6027. Since they are late B-type stars, none of the known three components in the HR 6026 system can be the source of the X-ray emission; this suggests the presence of an additional component in this system. In the case of the HR 6027 system, the spectroscopic companion $\mathrm{Ab}$ (with an estimated mass of $1 M_{\odot}$ ) is a good candidate for the source of the observed X-ray emission.

\footnotetext{
4 http://heasarc.gsfc.nasa.gov/docs/archive.html

5 http://heasarc.gsfc.nasa.gov/Tools/w3pimms.html
}

$\pi$ Sco: two ROSAT HRI observations are available for this source. We analyzed the longer one (Obs. Id. RH202044N00), with a $5100 \mathrm{~s}$ exposure time. The source is at an off-axis angle of $16.9^{\prime}$ near the edge of the detector. Source and background counts are extracted from circular regions of 50" and $80^{\prime \prime}$ radii, respectively. The net count rate for the source is $(4.4 \pm 0.3) \times$ $10^{-2}$ counts $\mathrm{s}^{-1}$. The intrinsic luminosity in the $[0.1-2.0] \mathrm{keV}$ range is then $\log \left[L_{X}\right] \sim 29.6$ and it suggests there is a late type, still unknown companion with $M \sim 0.4 M_{\odot}$.

$\tau$ Sco: this B0 V star has been thoroughly studied in X-rays (Mewe et al. 2003; Cohen et al. 1997). The analysis of the highresolution X-ray spectrum shows that the X-ray emission of this star can be explained by shocks in the strong radiation linedriven stellar wind of this hot star. In this case, the X-ray emission does not provide evidence of a late-type companion.

\section{Summary and conclusions}

We present new near-infrared interferometric data taken with AMBER at the VLTI of six B stars in the Upper Scorpius region. These observations are sensitive to companions between separations of $\sim 2$ mas and $\sim 100$ mas if the flux of the companion is at least a tenth of the flux of the primary (i.e., $\Delta K \lesssim 2.5$ ). This implies that we miss companions with masses $\leq 1 M_{\odot}$. We find a previously known companion around $\sigma$ Sco with a separation of $\sim 3$ mas and around half of the brightness of the primary. Furthermore, we detect the previously known Speckle companion around HR 6027.

Our analysis of archival X-ray data leads to detection of $\mathrm{X}$-ray emission from $\pi \mathrm{Sco}$ and $\tau \mathrm{Sco}$, as well as from the two subsystems of $v$ Sco, which are HR 6027 and HR 6026. For $\pi$ Sco and HR 6026, this observation strongly suggests the existence of an additional late-type companion. For the B0 star $\tau$ Sco the measured X-ray flux is due to shocks in the wind of the primary star itself. For HR 6027 the probable source of the X-ray emission is HR $6027 \mathrm{Ab}$.

Observations of these targets now exist over a complete range of spatial distances. However, all of the observational methods used have their own limitations. A very detailed modeling and discussion of the detection limits and their implications for the completeness of different surveys performed in Upper Scorpius can be found in Kouwenhoven et al. (2007). The adaptive optics surveys can detect companions for separations between $\sim 100$ mas and several tens of arcseconds, where the limiting flux ratio depends on the separation of the primary and secondary components. All of the targets discussed in our paper have been observed with the ADONIS near-infrared adaptive optics system and coronograph. The detection limit of these observations is $\sim 0.3^{\prime \prime}$ down to a flux ratio similar to our limiting flux ratio $(\Delta K=2.5 \mathrm{mag})$, and includes separations of up to $6.4^{\prime \prime}$ FOV. In general, the observations are sensitive to separations of down to $0.12^{\prime \prime}$ but only for companions with similar brightness to the primary. Five of our targets have also been observed with the adative optics system at the Gemini North telescope (Lafrenière et al. 2014). Here, too, the exact flux detection levels depend on the distance of the companions and the brightness of the primary star, but in general companions with separations between $\sim 0.1^{\prime \prime}$ and $\sim 5^{\prime \prime}$ can be detected. Thus, combining the adaptive optics and AMBER data we get a spatially complete sample that is limited by brightness and flux ratio.

In Table 2 we list our sample stars with the information about all of their known companions. Including the late type companions implied for $\pi$ Sco and HR 6026 in the X-ray data (see Sect. 5) we find $\sim 2.3$ companions per primary star on average. 
If we treat the systems of HR 6026 and HR 6027 as being independent instead of as one septuple system (since with separations of $41^{\prime \prime}$ and $20^{\prime \prime}$ they are are likely unbound) we find 1.9 companions per primary. These numbers are comparable with the average number of companions found for bright young stars in the Orion nebula cluster ( 1.5-2.5, Preibisch et al. 1999; Grellmann et al. 2013).

In the future, adaptive optics and interferometric observations with a higher dynamic range and a larger sample of stars in Upper Scorpius would be needed to perform meaningful statistics on the multiplicity in Upper Scorpius.

Acknowledgements. We gratefully acknowledge funding of this work by the German Deutsche Forschungsgemeinschaft, DFG project number PR 569/8-1. This research has made use of the Jean-Marie Mariotti Center AMBER data reduction package ${ }^{6}$ and LITpro $^{7}$ service co-developed by CRAL, LAOG and FIZEAU. This research has made use of the SIMBAD database, operated at CDS, Strasbourg, France. We thank the anonymous referee for patiently reading the paper several times and for his/her suggestions to improve the manuscript.

\section{References}

Andersen, J., \& Nordstrom, B. 1983, A\&AS, 52, 471

Bate, M. R. 2009, MNRAS, 397, 232

Batten, A. H., Fletcher, J. M., \& MacCarthy, D. G. 1989, Publications of the Dominion Astrophysical Observatory Victoria, 17, 1

Blaauw, A. 1991, in The Physics of Star Formation and Early Stellar Evolution, eds. C. J. Lada, \& N. D. Kylafis, NATO ASIC Proc., 342, 125

Chelli, A., Utrera, O. H., \& Duvert, G. 2009, A\&A, 502, 705

Cohen, D. H., Cassinelli, J. P., \& Waldron, W. L. 1997, ApJ, 488, 397

de Bruijne, J. H. J. 1999, MNRAS, 310, 585

de Zeeuw, P. T., Hoogerwerf, R., de Bruijne, J. H. J., Brown, A. G. A., \& Blaauw, A. 1999, AJ, 117, 354

Delgado-Donate, E. J., Clarke, C. J., Bate, M. R., \& Hodgkin, S. T. 2004, MNRAS, 351, 617

Duchêne, G., \& Kraus, A. 2013, ARA\&A, 51, 269

Eggleton, P. P., \& Tokovinin, A. A. 2008, MNRAS, 389, 869

Evans, N. R., DeGioia-Eastwood, K., Gagné, M., et al. 2011, ApJS, 194, 13

Favata, F., \& Micela, G. 2003, Space Sci. Rev., 108, 577

Goodwin, S. P., Kroupa, P., Goodman, A., \& Burkert, A. 2007, Protostars and Planets V (Tucson: University of Arizona Press), 591, 133
Grellmann, R., Preibisch, T., Ratzka, T., et al. 2013, A\&A, 550, A82

Güdel, M., \& Nazé, Y. 2009, A\&ARv, 17, 309

Harmanec, P. 1987, Bull. Astron. Institutes of Czechoslovakia, 38, 283

Kouwenhoven, M. B. N., Brown, A. G. A., Zinnecker, H., Kaper, L., \& Portegies Zwart, S. F. 2005, A\&A, 430, 137

Kouwenhoven, M. B. N., Brown, A. G. A., Portegies Zwart, S. F., \& Kaper, L. 2007, A\&A, 474, 77

Kudritzki, R.-P., \& Puls, J. 2000, ARA\&A, 38, 613

Lafrenière, D., Jayawardhana, R., van Kerkwijk, M. H., Brandeker, A., \& Janson, M. 2014, ApJ, 785, 47

Levato, H., Malaroda, S., Morrell, N., \& Solivella, G. 1987, ApJS, 64, 487

Mathieu, R. D., \& Zinnecker, H. 2000, PASP, 112, 1512

McAlister, H., Hartkopf, W. I., \& Franz, O. G. 1990, AJ, 99, 965

Mewe, R., Raassen, A. J. J., Cassinelli, J. P., et al. 2003, Adv. Space Res., 32, 1167

North, J. R., Davis, J., Tuthill, P. G., Tango, W. J., \& Robertson, J. G. 2007, MNRAS, 380, 1276

Petrov, R. G., Malbet, F., Weigelt, G., et al. 2007, A\&A, 464, 1

Preibisch, T., \& Mamajek, E. 2008, The Nearest OB Association: ScorpiusCentaurus (Sco OB2), ed. B. Reipurth, 235

Preibisch, T., \& Zinnecker, H. 1999, AJ, 117, 2381

Preibisch, T., Balega, Y., Hofmann, K.-H., Weigelt, G., \& Zinnecker, H. 1999, New Astron., 4, 531

Preibisch, T., Weigelt, G., \& Zinnecker, H. 2001, in The Formation of Binary Stars, eds. H. Zinnecker, \& R. Mathieu, IAU Symp., 200, 69

Preibisch, T., Brown, A. G. A., Bridges, T., Guenther, E., \& Zinnecker, H. 2002, AJ, 124, 404

Preibisch, T., Kim, Y.-C., Favata, F., et al. 2005, ApJS, 160, 401

Rizzuto, A. C., Ireland, M. J., Robertson, J. G., et al. 2013, MNRAS, 436, 1694

Schmitt, J. H. M. M., Golub, L., Harnden, Jr., F. R., et al. 1985, ApJ, 290, 307

Schmitt, J. H. M. M., Kahabka, P., Stauffer, J., \& Piters, A. J. M. 1993, A\&A, 277,114

Siess, L., Dufour, E., \& Forestini, M. 2000, A\&A, 358, 593

Slesnick, C. L., Hillenbrand, L. A., \& Carpenter, J. M. 2008, ApJ, 688, 377

Stelzer, B., Huélamo, N., Hubrig, S., et al. 2005, in 13th Cambridge Workshop on Cool Stars, Stellar Systems and the Sun, eds. F. Favata, G. A. J. Hussain, \& B. Battrick, ESA SP, 560, 213

Stelzer, B., Micela, G., Hamaguchi, K., \& Schmitt, J. H. M. M. 2006, A\&A, 457, 223

Tallon-Bosc, I., Tallon, M., Thiébaut, E., et al. 2008, in SPIE Conf. Ser., 7013

Tatulli, E., Millour, F., Chelli, A., et al. 2007, A\&A, 464, 29

Tokovinin, A. A. 1997, A\&AS, 124, 75

Townsley, L. K., Broos, P. S., Corcoran, M. F., et al. 2011, ApJS, 194, 1

Zinnecker, H., \& Yorke, H. W. 2007, ARA\&A, 45, 481

Pages 8 to 11 are available in the electronic edition of the journal at http://www . aanda.org

\footnotetext{
6 Available at http://www.jmmc.fr/amberdrs

7 LITpro software available at http://www.jmmc. fr/litpro
} 


\section{Appendix A: Additional material}
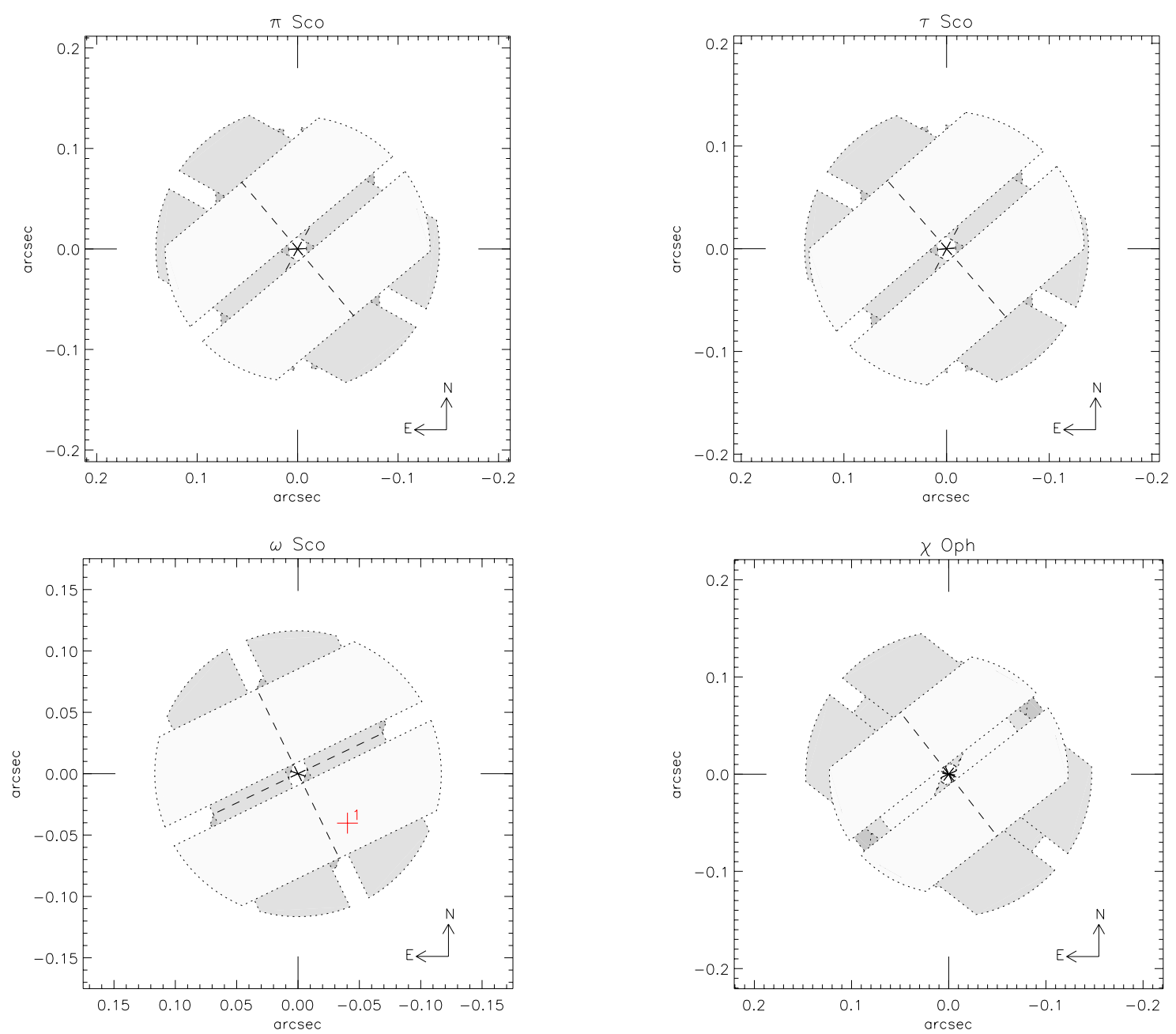

Fig. A.1. Coverage of the area around the observed objects, where a binary component within the defined conditions (i.e., $\Delta K=2.5$, see Chap. 3 ) can be excluded from the AMBER data. The different colors indicate the areas for the three different baselines and position angles. Upper left: $\pi$ Sco. Upper right: $\tau$ Sco. Lower left: $\omega$ Sco. Lower right: $\chi$ Oph.

Table A.1. Properties of the septuple $v$ Sco.

\begin{tabular}{lccccc}
\hline \hline Star & $\begin{array}{c}\text { Sep./period of component } \\
{\left[{ }^{\prime \prime}\right] / \text { days }}\end{array}$ & $\begin{array}{c}\text { Bright. }(V) \\
{[\mathrm{mag}]}\end{array}$ & $\begin{array}{c}\text { SpT } \\
\begin{array}{c}\text { Mass } \\
{\left[M_{\odot}\right]}\end{array}\end{array}$ & Reference \\
\hline HR 6027 Aa Primary & & 4.37 & B2IV & 10 & 1 \\
HR 6027 Ab Spectr. & $5.55 \mathrm{~d}$ & 6.9 & & 1 & $1,2,3,6$ \\
HR 6027 B Visual 1 & $1.305^{\prime \prime}$ & 5.4 & & 6 & $1,2,6$ \\
HR 6027 C Visual 2 & $0.063^{\prime \prime}$ & 6.6 & & 6 & 1,4 \\
HR 6026 A Primary & $41.1^{\prime \prime}$ & 6.9 & B8V & 3 & 1 \\
HR 6026 Ba Visual & $2.0^{\prime \prime}$ & 7.4 & B9V & 2.7 & 1,2 \\
HR 6026 Bb Spectr. & & 7.9 & B9V & 2.7 & 1,5 \\
HR 6026 C X-ray & & & & $\sim 1.5$ & see Sect. 5 \\
\hline
\end{tabular}

References. (1) MSC, Tokovinin (1997); (2) Levato et al. (1987); (3) Batten et al. (1989); (4) McAlister et al. (1990); (5) Andersen \& Nordstrom (1983); (6) Kouwenhoven et al. (2007). 
R. Grellmann et al.: NIR-interferometry in Upper Scorpius
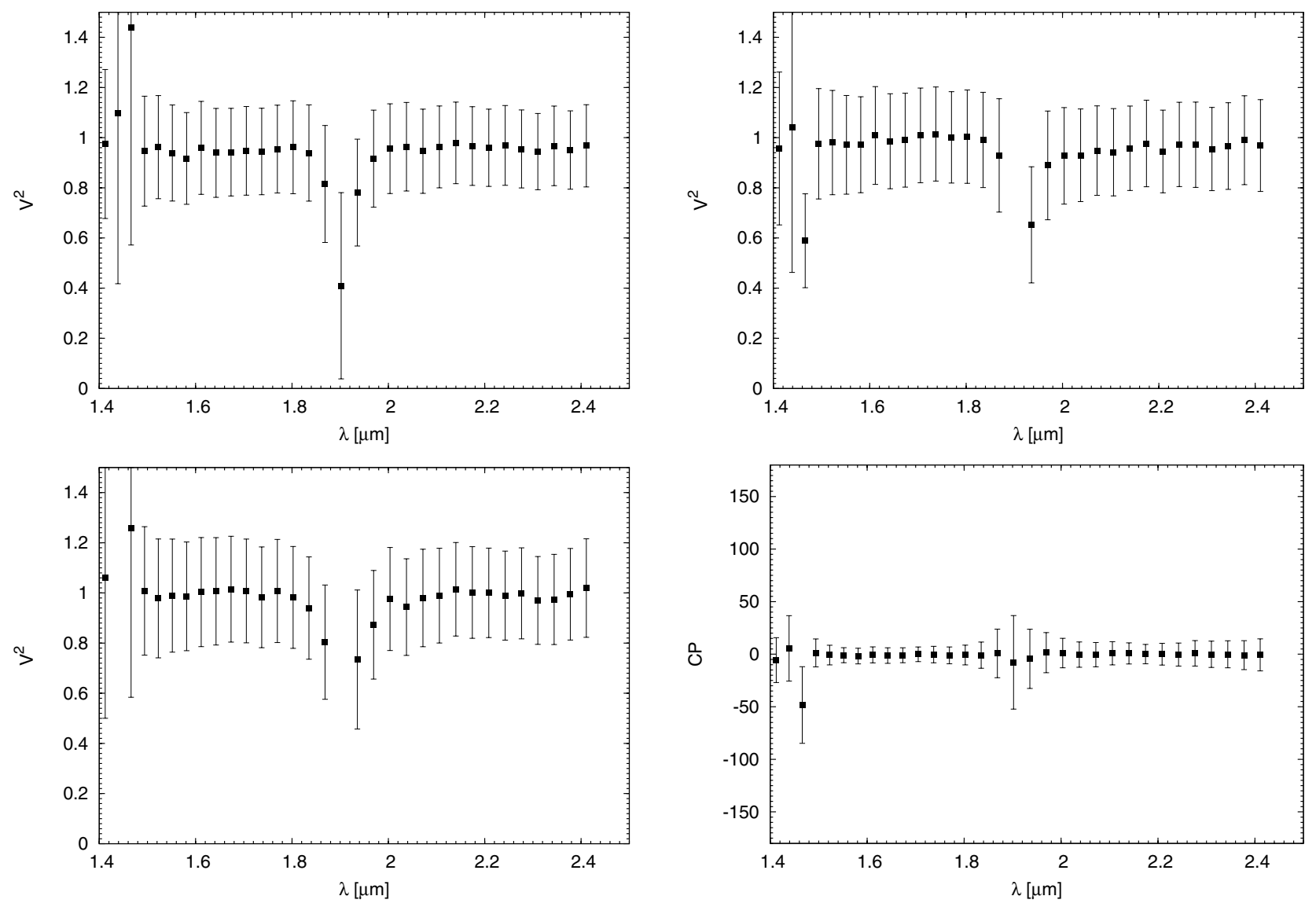

Fig. A.2. Visibilities and closure phase of $\pi$ Sco versus wavelength for the $H$ and $K$ bands measured with AMBER on May 6, 2010.
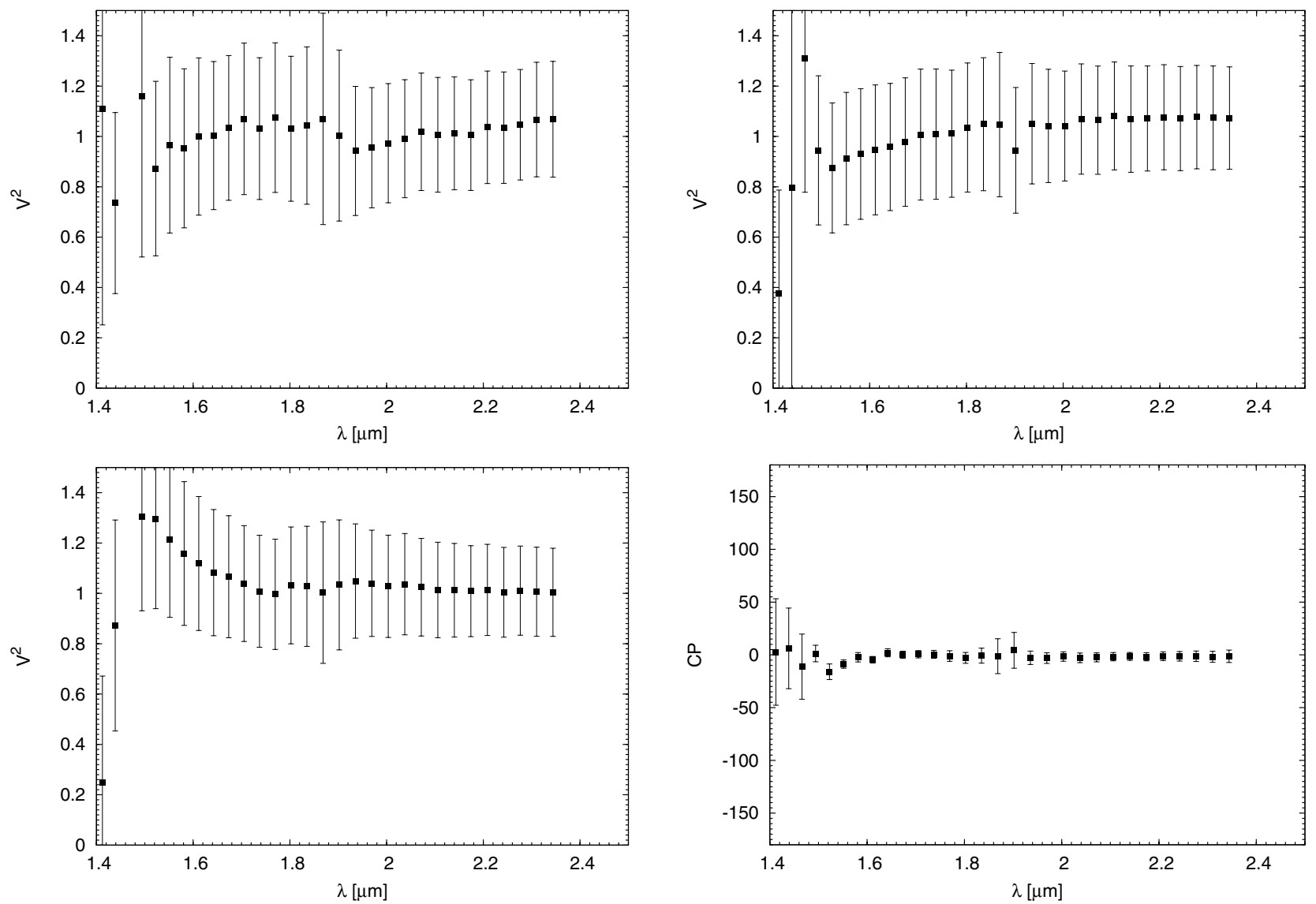

Fig. A.3. Visibilities and closure phase of $\pi$ Sco versus wavelength for the $H$ and $K$ bands measured with AMBER on May 10, 2010. 
A\&A 578, A84 (2015)
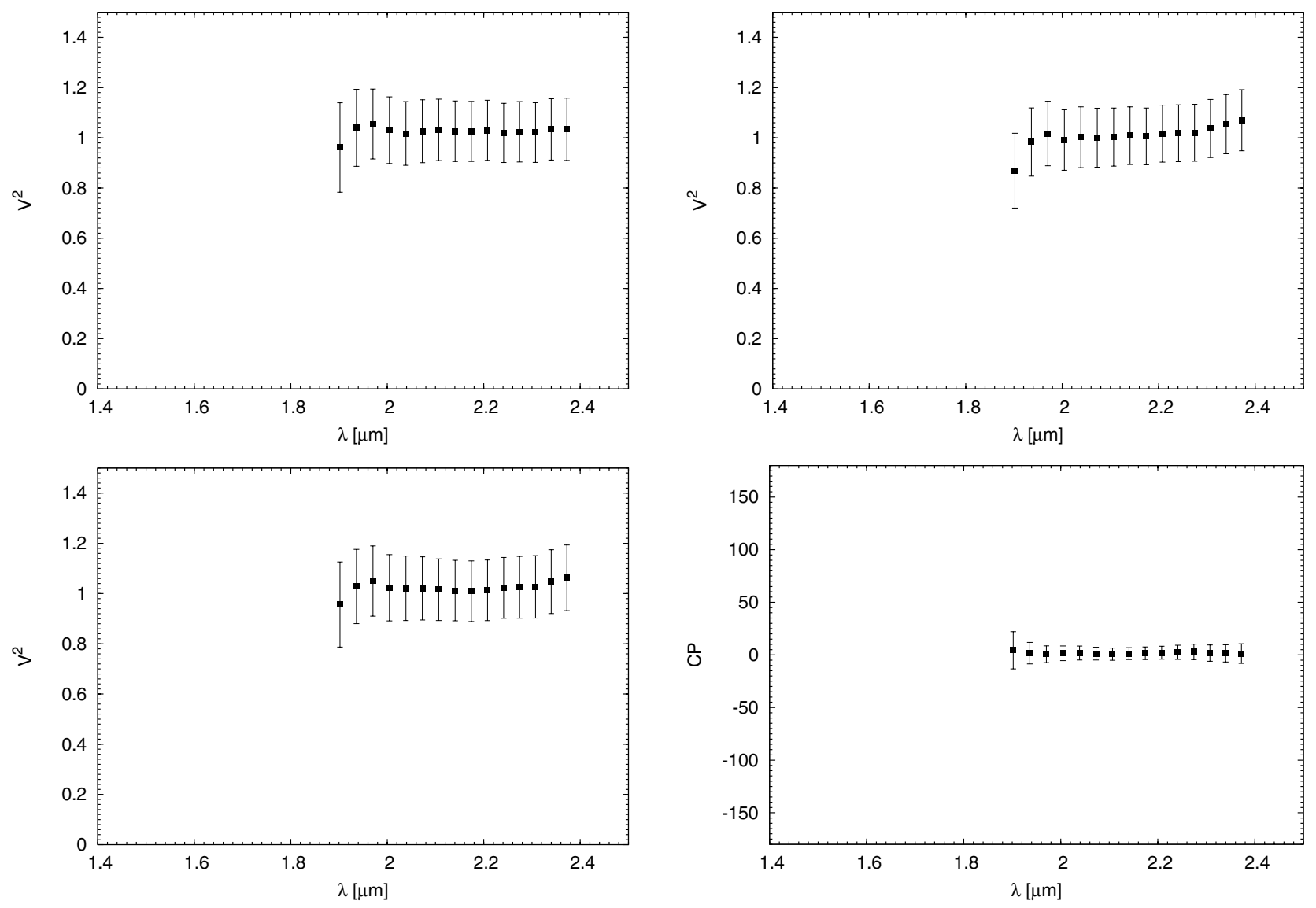

Fig. A.4. Visibilities and closure phase of $\omega$ Sco versus wavelength for the $H$ and $K$ bands measured with AMBER on May 11, 2010.
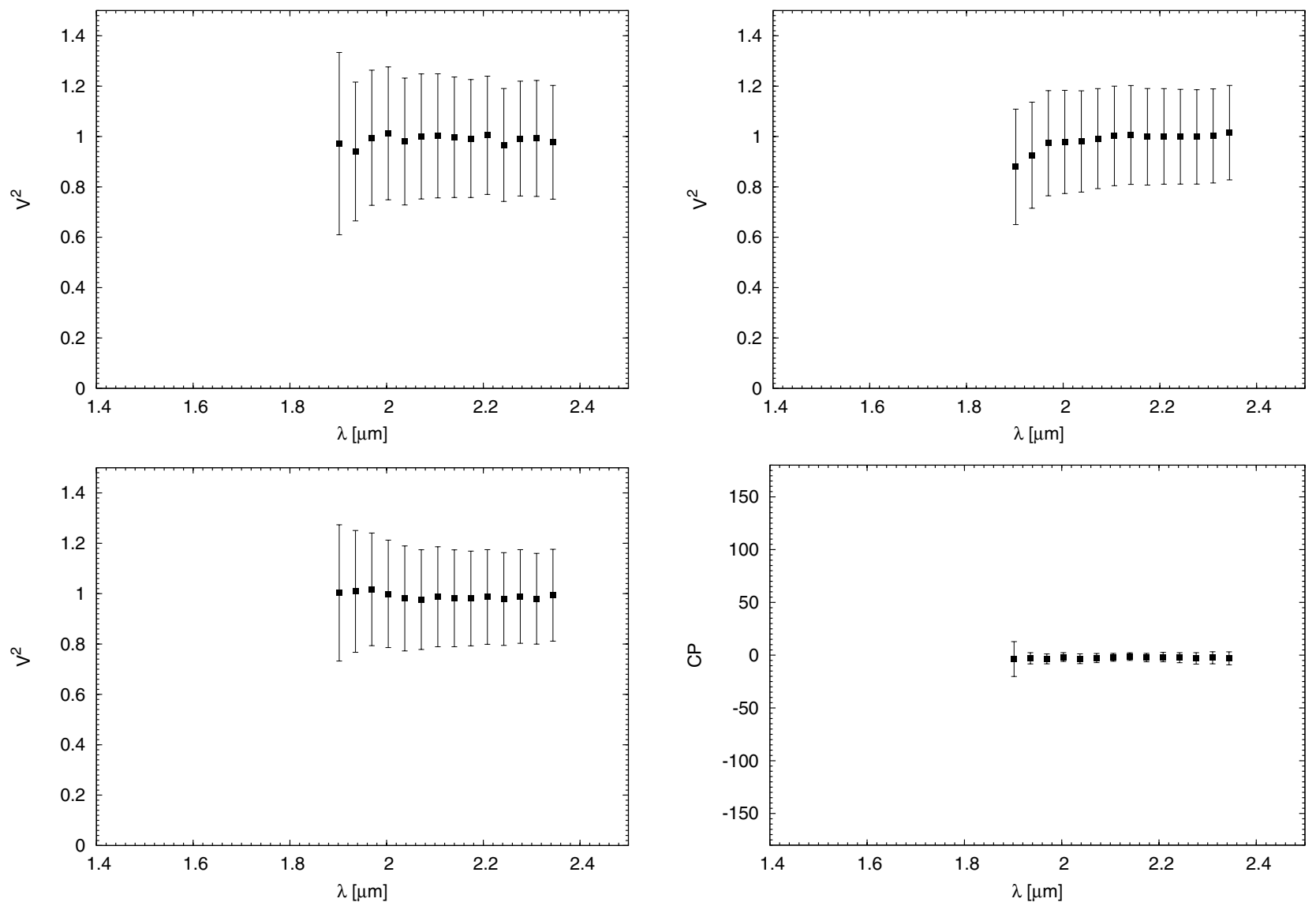

Fig. A.5. Visibilities and closure phase of $\tau$ Sco versus wavelength for the $H$ and $K$ bands measured with AMBER on May 10, 2010. 
R. Grellmann et al.: NIR-interferometry in Upper Scorpius
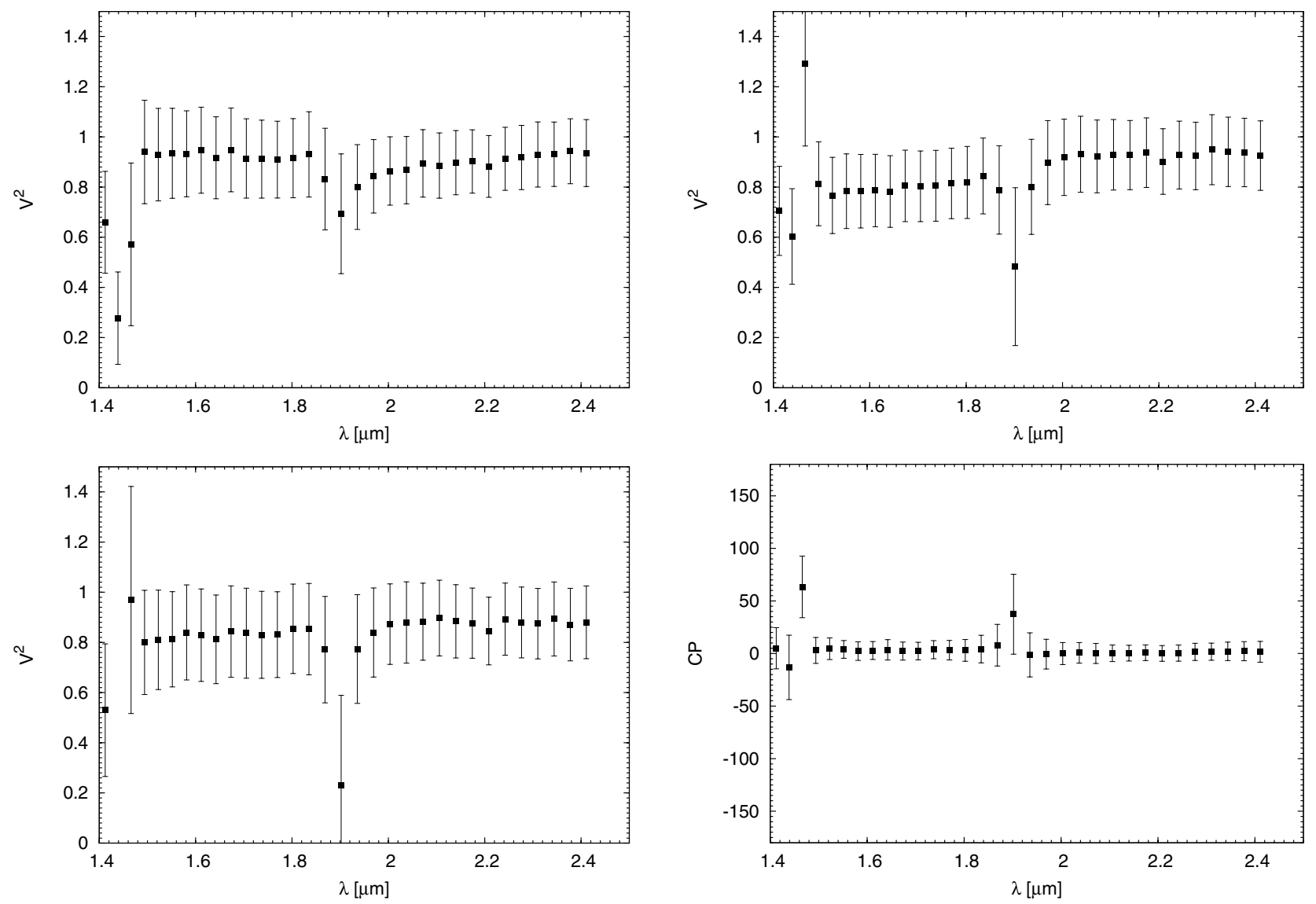

Fig. A.6. Visibilities and closure phase of $\chi$ Oph versus wavelength for the $H$ and $K$ bands measured with AMBER on May 6, 2010.
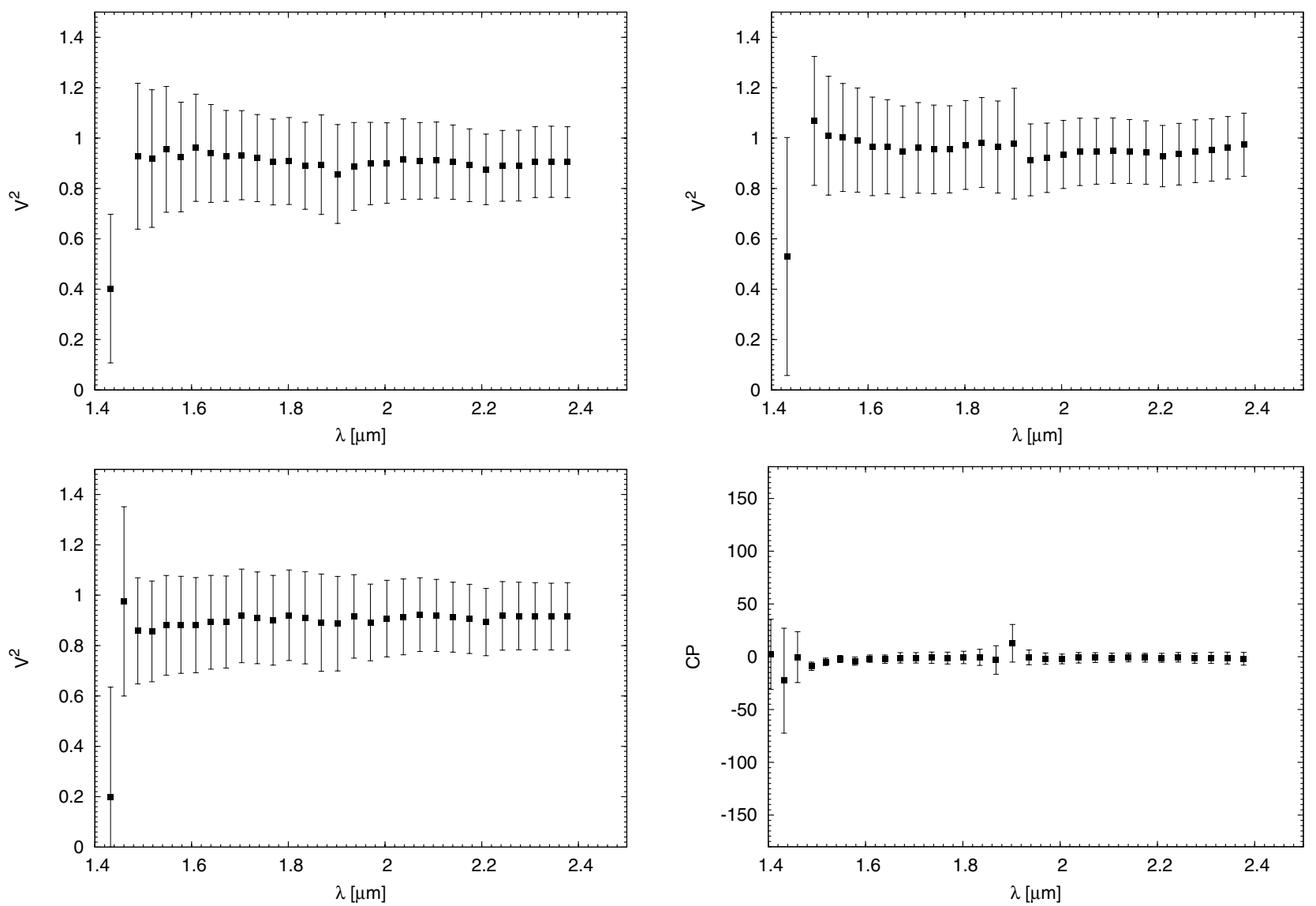

Fig. A.7. Visibilities and closure phase of $\chi$ Oph versus wavelength for the $H$ and $K$ bands measured with AMBER on May 11, 2010. 